Victorino Farga $C$.

\title{
Tuberculosis en grupos de riesgo en la Región Metropolitana. 2008
}

\author{
CHRISTIAN GARCÍA C.*
}

Tuberculosis in risk groups within Region Metropolitana, Chile, 2008

Several risk factors have been identified of becoming ill of tuberculosis. This article estimates the importance of risk groups associated with tuberculosis within the Metropolitan Region of Chile in 2008. The incidence rate of tuberculosis for the Metropolitan Region was 14.19 per 100.000 inhabitants, being much higher in people with HIV (380,5), in homeless people (218. 7), in convicts (213.4), contacts of tuberculosis patients (104.5), foreigners (37.5) and in elderly people (32.8). All of these risk groups have a significant association with tuberculosis and their odds ratios ranged from 29 (HIV) to 2.6 (elderly people). In spite of being few cases, the risk of becoming ill of tuberculosis in people belonging to risk groups such as HIV, homeless, convicts and foreigners is higher in comparison with the rest of the population. The elderly people also have a risk higher than the rest of population, representing $18.8 \%$ of the tuberculosis cases in the region.

Key words: Tuberculosis, incidence, risk groups, HIV, homeless, convict, contact of tuberculosis, foreigners, elderly people.

\section{Resumen}

Se han identificado diversos factores de riesgo de enfermar de tuberculosis. Este artículo estima la importancia de los grupos de riesgo asociados a tuberculosis dentro de la Región Metropolitana de Chile en 2008. La tasa de incidencia de tuberculosis para la Región Metropolitana fue de 14,19 por cien mil habitantes, siendo mayor en las personas con VIH (380,5), en personas en situación de calle $(218,7)$, reos $(213,4)$, contactos de pacientes con tuberculosis $(104,5)$, en extranjeros $(37,5) y$ en adultos mayores $(32,8)$. Todos ellos con asociación significativa y odds ratio entre 29 (VIH) y 2,6 (adultos mayores). A pesar de ser pocos casos, el riesgo de enfermar de tuberculosis en personas con $V I H$, personas en situación de calle, reos y extranjeros es elevado en comparación con el resto de la población. También con un riesgo elevado se encuentran los adultos mayores, que representan el 18,8\% de las personas con tuberculosis en la región.

Palabras clave: Tuberculosis, incidencia, factores de riesgo, VIH, situación de calle, reo, contactos de tuberculosis, extranjero, adulto mayor.

La tuberculosis persiste como un problema de salud pública a nivel mundial. Se estima que en el año 2007 había 13,7 millones de casos prevalentes; 9,27 millones de casos nuevos y 1,3 millones de muertes por esta enfermedad ${ }^{1}$.

A fines del siglo pasado se asistió a un resurgimiento de la tuberculosis en países desarrollados y en desarrollo, en parte por la pandemia de SIDA; también por el aumento de las poblaciones vulnerables y de migraciones derivadas de gue- rras y hambrunas, pero en su mayor parte, por el descuido en que cayeron los programas de control en la mayoría de los países ${ }^{2,3}$. Para afrontar este problema los objetivos de Desarrollo del Milenio de la ONU han establecido en sus metas para el 2015 reducir a la mitad la incidencia de la tuberculosis encontrada en $1990^{4}$. Esto ha significado un nuevo impulso al control de esta enfermedad en el mundo, con estímulos a la investigación en medicamentos, nuevos métodos diagnósticos y

* Programa de Tuberculosis. Ministerio de Salud. Chile. 
vacunas, y fondos para avanzar hacia el control de la enfermedad.

Cientos de muertes y miles de enfermos se presentan anualmente en Chile. Entre los años 1997 y 2007 murieron 2.739 personas de tuberculosis, es decir una muerte por cada día hábil, con una tasa de mortalidad específica para el período de 1,7 por 100.000 habitantes.

Chile ha mantenido una reducción permanente de la tasa de incidencia de casos nuevos en todas sus formas: desde 78 casos por 100.000 habitantes en 1980, a 13,6 en 2008, encontrándose actualmente por debajo del umbral de la etapa de eliminación de la tuberculosis (tasa $<20 / 100.000$ ) y progresando hacia la etapa de eliminación avanzada (tasa $<10 / 100.000$ ). Esto ubica a Chile entre los países de América Latina denominados de baja prevalencia. La distribución por edades y sexo sigue el modelo de países desarrollados, con mayor proporción de casos y mayores tasas desplazadas a edades más avanzadas, con una creciente razón masculino/femenino ${ }^{5}$.

El desafío principal para Chile es seguir reduciendo la tasa de incidencia de la enfermedad, lo que se hace cada vez más difícil. La mantención de las actividades de localización de casos dentro de los sintomáticos respiratorios es la medida más importante para no revivir la experiencia del resurgimiento violento de esta enfermedad a fines del siglo pasado en países con tasas incluso menores a las que nuestro país tiene hoy ${ }^{2,3}$.

Identificar y focalizar la pesquisa de casos en los grupos con mayor riesgo, sin dejar de lado las actividades habituales de localización pasiva en los consultantes espontáneos a los Servicios de Salud, es una tarea vital para mantener y acelerar la reducción de la endemia tuberculosa.

El riesgo de infección por Mycobacterium tuberculosis está dado principalmente por el número de enfermos contagiantes que haya en la comunidad. Los condicionantes de hacinamiento, tamaño familiar y período de contagiosidad, son factores secundarios que llevan a una población a tener mayor cercanía con un enfermo contagioso, con el consiguiente riesgo de exposición. Son los contactos más próximos a enfermos con tuberculosis pulmonar con baciloscopía positiva, los que tienen mayor riesgo de ser infectados. $\mathrm{La}$ proporción de personas infectadas aumenta a mayor edad, igual que la diferencia hombre/mujer, población urbana/rural, estratos socioeconómicos bajos y condiciones de hacinamiento.

El riesgo de enfermar depende en primera instancia de la exposición y del riesgo de infección. Se ha aceptado como regla general que una persona infectada tiene un $10 \%$ de riesgo de enfermar de tuberculosis en toda su vida, la mitad dentro de los primeros 5 años de ocurrida la infección y la otra mitad posteriormente. Este riesgo contrasta con el de las personas infectadas con el VIH en los que varía entre 5-15\% anual. A mayor edad también aumenta el riesgo de enfermar, por dos razones fundamentales: mayor prevalencia de la infección por aumento acumulativo de la prevalencia en el tiempo y reducción paulatina de la inmunidad, lo que conduce a un mayor riesgo a partir de los 60 años. La respuesta inmunitaria del huésped determinará, en última instancia, la posibilidad de enfermar o de eliminar al bacilo después de haber sido infectado ${ }^{6}$.

Se ha observado que existen grupos especiales con características desfavorables que determinan un mayor riesgo de enfermar de tuberculosis. Son grupos con factores de riesgo (FR) variables, entre los que destacan los adultos mayores (principal factor de riesgo en el país), las personas privadas de libertad, individuos en situación de calle, indigentes y personas con VIH/SIDA ${ }^{3,7-9}$.

La Región Metropolitana (RM), tiene el 40\% de la población de Chile; es la región más numerosa, donde se concentra históricamente un porcentaje similar de enfermos que en el resto del país. El objetivo de este trabajo es estimar la importancia de cada uno de los grupos de riesgo asociados a la tuberculosis dentro de la RM, su tasa de incidencia, su asociación con la enfermedad y la magnitud del riesgo, comparada con la población sin FR.

\section{Metodología}

El Programa Nacional de Control de la Tuberculosis (PNT), tiene un sistema de registros estandarizado para todo el país, donde cada servicio de salud informa la edad, el diagnóstico, método de confirmación, información territorial y, sistemáticamente desde el año 2008, la presencia de FR asociados. A partir de los registros de los 6 Servicios de Salud de la RM, se conforma el registro de la Secretaría Regional Ministerial (SEREMI) de Salud, desde la cual se obtuvieron los casos diagnosticados con tuberculosis y los FR encontrados durante el año 2008.

Se evaluaron los siguientes grupos con factores de riesgo: adulto mayor (65 años o más); infectado VIH/SIDA; contacto de tuberculosis; drogadicción; extranjero (referido a haber nacido fuera de Chile); funcionario de Salud; persona en situación de calle (indigente); alcoholismo, y sujeto privado de libertad (reo). La base obtenida se comparó con las notificaciones obligatorias 
registradas en la SEREMI para ese año y con las bases de datos del Nivel Central del Ministerio de Salud, para validar la información.

Se calculó la prevalencia de enfermos para la población de la RM como la totalidad de casos notificados como residentes en el año 2008 por cada 100.000 habitantes, y la tasa de incidencia como los casos nuevos notificados como residentes en la RM por 100.000 habitantes.

El número de casos correspondientes a cada FR se obtuvo del registro elaborado en la SEREMI una vez validado. Para estimar la prevalencia y la incidencia se calcularon los numeradores para los distintos grupos de riesgo basándose en información secundaria y metodologías de estimación publicadas. La Tabla 1 muestra la fuente de información y el método de cálculo de cada una de las poblaciones estimadas para los numeradores.

No se consideró para estos cálculos la presencia de más de un FR por individuo; por lo tanto, un paciente con más de un FR estaría aportando a las tasas de cada uno de ellos.

El cálculo de la población con al menos un FR se realizó sumando los estimados de población de todos los factores analizados y se asumió una superposición de un $15 \%$ de FR en la población general de la RM, siendo la población estimada de personas con al menos un FR el $85 \%$ de la suma aritmética de las poblaciones estimadas para la región de cada uno de los FR por separado.

Se comparó la incidencia para cada grupo de

Tabla 1. Fuentes, métodos y población estimada en la RM por factor de riesgo

\begin{tabular}{|c|c|c|c|}
\hline Factor de Riesgo & Fuente de información & Método de Cálculo & $\begin{array}{c}\text { Población Estimada } \\
\text { en la RM } 2008\end{array}$ \\
\hline Adulto Mayor & $\begin{array}{l}\text { Instituto Nacional de Esta- } \\
\text { dísticas (INE), Proyección de } \\
\text { población www.ine.cl. }\end{array}$ & $\begin{array}{l}\text { Población estimada de } 65 \text { años y } \\
\text { más en la RM }\end{array}$ & 563.565 \\
\hline VIH & $\begin{array}{l}\text { Diagnóstico VIH/SIDA Re- } \\
\text { gión Metropolitana }^{10}\end{array}$ & $\begin{array}{l}\text { CONASIDA; Panorama epidemio- } \\
\text { lógico de la infección por } \\
\text { VIH/SIDA en Chile - Año } 2001^{11}\end{array}$ & 18.396 \\
\hline Contacto & $\begin{array}{l}\text { Registro de casos TBC Re- } \\
\text { gión Metropolitana }\end{array}$ & $\begin{array}{l}\text { Casos de TBC pulmonar Baci- } \\
\text { loscopía positiva } \times 4 \text { contactos } \\
\text { promedio }\end{array}$ & 3.828 \\
\hline Drogas & $\begin{array}{l}\text { 1. Séptimo estudio nacional } \\
\text { de drogas en población gene- } \\
\text { ral de Chile } 2006^{12} \\
\text { 2. INE }\end{array}$ & $\begin{array}{l}\text { (Proporción de personas de } 12 \text { - } \\
64 \text { años del } 2006 \text { con consumo } \\
\text { de cocaína en todas sus formas, } \\
\text { más proporción de personas con } \\
\text { dependencia a marihuana en los } \\
\text { consumidores prevalentes de la } \\
\text { RM) x (población de } 12 \text { a } 64 \text { años } \\
\text { de la RM de 2008) }\end{array}$ & 166.631 \\
\hline Extranjero & $\begin{array}{l}\text { Informe estadístico Estima- } \\
\text { ción de población extranjeros } \\
\text { a diciembre de } 2008^{13}\end{array}$ & $\begin{array}{l}\text { Número estimado de extranjeros } \\
\text { RM a Diciembre } 2008\end{array}$ & 202.685 \\
\hline $\begin{array}{l}\text { Funcionario } \\
\text { de Salud }\end{array}$ & $\begin{array}{l}\text { Observatorio de Recursos } \\
\text { Humanos }^{14}\end{array}$ & $\begin{array}{l}90.000 \text { en SNSS país } 2004 \text {, esti- } \\
\text { mando aumento } 3 \% \text { en la dotación } \\
\text { anual y } 60 \% \text { de los funcionarios } \\
\text { dentro de la RM }\end{array}$ & 60.777 \\
\hline $\begin{array}{l}\text { Personas en } \\
\text { situación de calle }\end{array}$ & $\begin{array}{l}\text { 1. Catastro nacional de perso- } \\
\text { nas en situación de calle }{ }^{15} \\
\text { 2. INE }\end{array}$ & $\begin{array}{l}\text { Tasa } 6,1 / 10.000 \text { aplicada a la po- } \\
\text { blación RM } 2008\end{array}$ & 4.115 \\
\hline Alcoholismo & $\begin{array}{l}\text { 1. Séptimo estudio nacional } \\
\text { de drogas en población gene- } \\
\text { ral de Chile } 2006^{12} \\
\text { 2. INE }\end{array}$ & $\begin{array}{l}\text { (Proporción de personas con abuso } \\
\text { de alcohol en la RM en personas } \\
\text { de } 12-64 \text { años) x (población 12- } \\
64 \text { de la RM. 2008) }\end{array}$ & 355.177 \\
\hline $\begin{array}{l}\text { Personas privadas } \\
\text { de libertad }\end{array}$ & Página web de Gendarmería ${ }^{16}$ & $\begin{array}{l}\text { Personas en prisión en sistema } \\
\text { cerrado en la RM a diciembre de } \\
\text { año } 2009\end{array}$ & 19.936 \\
\hline
\end{tabular}


riesgo por separado, para el total de la población, para la población sin FR y para la población con al menos un FR. Para establecer asociaciones entre cada factor y el diagnóstico de tuberculosis se utilizó la prueba de asociación $\chi^{2}$ y posteriormente se calculó el Odds Ratio para cada FR, cuyo intervalo de confianza fue calculado con el método de Woolf.

\section{Resultados}

El número total de casos notificados de tuberculosis en la Región Metropolitana el año 2008 alcanzó a 1.033. Casos antes tratados: 76 (7,3\%); Casos nuevos: 957 (92,7\% del total de notificados). La proporción de casos por sexo es similar en estos tres grupos variando entre $66-68 \%$ de sexo masculino y $32-34 \%$ de sexo femenino (Tabla 2).

Tabla 2. Número y proporción de casos de tuberculosis en la RM. 2008

\begin{tabular}{|lcccccc|}
\hline & \multicolumn{2}{c}{ Notificados } & \multicolumn{2}{c|}{ Casos nuevos } & \multicolumn{2}{c|}{$\begin{array}{c}\text { Casos antes } \\
\text { tratados }\end{array}$} \\
& n & \% & n & $\%$ & n & \% \\
Casos & 1.033 & 100 & 957 & 92,7 & 76 & 7,3 \\
Sexo & & & & & & \\
Femenino & 348 & 34 & 324 & 34 & 24 & 32 \\
Masculino & 685 & 66 & 633 & 66 & 52 & 68 \\
\hline
\end{tabular}

Tabla 3. Edad por Factores de Riesgo, RM. 2008

\begin{tabular}{|lcclll|}
\hline & $\mathbf{n}$ & $\mathbf{\%}$ & $\begin{array}{c}\text { Promedio } \\
\text { edad } \\
\text { (años) }\end{array}$ & IC 95\% \\
\hline Total casos nuevos & 957 & 100 & 46 & 44,5 & $-46,9$ \\
Femenino & 324 & 34 & 46,7 & 44,5 & $-48,9$ \\
Masculino & 633 & 66 & 45,3 & 43,8 & $-46,7$ \\
Al menos un FR & 396 & 41,4 & 52,38 & $50,18-54,58$ \\
Femenino & 127 & 32 & 56,6 & 52,5 & $-60,6$ \\
Masculino & 269 & 68 & 50,3 & 47,7 & $-52,9$ \\
Al menos un FR* & 211 & - & 33,9 & 32,4 & $-35,5$ \\
Femenino & 53 & - & 32 & 28,6 & $-35,3$ \\
Masculino & 158 & - & 34,6 & 32,9 & $-36,3$ \\
Sin FR & 561 & 58,6 & 41,2 & 39,9 & $-42,3$ \\
Femenino & 197 & 35,1 & 40,4 & 38,3 & $-42,4$ \\
Masculino & 364 & 64,8 & 41,6 & 40,1 & $-43,0$ \\
\hline
\end{tabular}

*Descartando a los adultos mayores. FR: factor de riesgo.
Los casos nuevos tienen una media de edad de 46 años (IC $95 \%$ 44,5-46,9), sin diferencias significativas entre las medias de ambos sexos, femenino 46,7 años (IC 95\% 44,5-48,9) y masculino 45,3 años (IC 43,8-46,7). Tampoco se observan diferencias entre los promedios de edad entre sexos en las poblaciones sin FR $\mathrm{y}$ en las con por lo menos uno de ellos.

Diferencias significativas se encuentran entre la edad del grupo con por lo menos un FR $y$ sin FR, siendo mayor en el primero para el total y para cada sexo, principalmente por tomar como FR ser mayor de 65 años. Si se descarta el grupo de personas mayores de 65 años y se compara la edad promedio entre los casos sin FR y los restantes con al menos un FR, se obtiene una media de edad para el grupo con al menos un FR de 33,9 años (IC95\% 32,4-35,5), para los de sexo masculino 34,6 años (IC95\% 32,9-36,3) y para los de sexo femenino 32,0 años (IC95\% 28,6-35,3). La edad de este grupo es significativamente menor que los que no tienen FR, lo mismo para cada sexo (Tabla 3).

En los casos nuevos, 396 $(41,3 \%)$ presentaron al menos un FR. En la Tabla 4 y en la Figura 1 se resumen los casos con factores de riesgo y las tasas de incidencia específicas.

Tener al menos un FR significaría tener una tasa de incidencia de tuberculosis de 33,4 por 100.000 habitantes. Es decir 2,4 veces mayor que la de toda la población de la RM (con y sin FR) y de 3,4 veces más que la de las personas que no tienen ningún FR registrado.

Seis de los 9 FR presentaron tasas mayores de tuberculosis que el total de población de la RM: adultos mayores, infectados VIH, contactos, extranjeros, situación de calle y reos. Por otro lado, los siguientes tres FR presentaron 


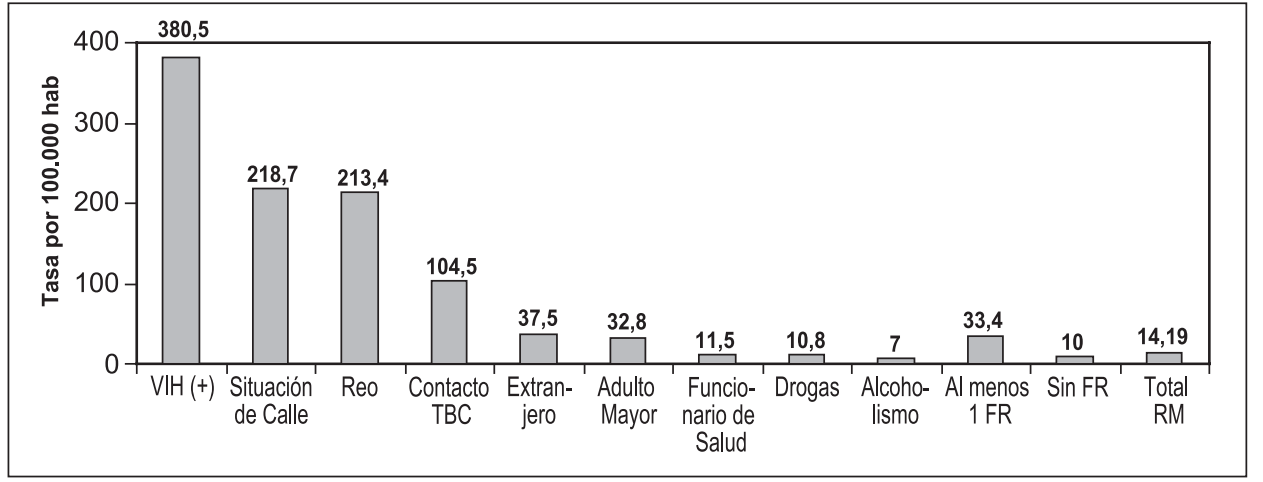

Figura 1. Tasa de incidencia de tuberculosis por factor de riesgo (FR). Región Metropolitana 2008.

Tabla 4. Pacientes y tasa de incidencia por factor de riesgo RM. 2008

\begin{tabular}{|lrrc|}
\hline Factor de Riesgo & \multicolumn{1}{c}{$\mathbf{n}$} & Población & Tasa x 100.000 \\
\hline Adulto mayor & 185 & 563.565 & 32,8 \\
\hline VIH (+) & 70 & 18.396 & 380,5 \\
\hline Contacto TBC & 4 & 3.828 & 104,5 \\
\hline Drogadicción & 18 & 166.631 & 10,8 \\
\hline Extranjero & 76 & 202.685 & 37,5 \\
Funcionario de salud & 7 & 60.777 & 11,5 \\
\hline Situación de calle & 9 & 4.115 & 218,7 \\
\hline Alcoholismo & 25 & 355.177 & 7,0 \\
\hline Reo & 39 & 18.272 & 213,4 \\
\hline Al menos 1 FR & 396 & 1.184 .688 & 33,4 \\
\hline Sin FR & 561 & 5.5604 .13 & 10 \\
\hline Total casos nuevos & 957 & 6.745 .651 & 14,19 \\
\hline
\end{tabular}

FR: factor de riesgo.

Tabla 5. Odds ratio (OR) por factor de riesgo, RM. 2008

\begin{tabular}{|lrrrc|}
\hline Factor de Riesgo & OR & \multicolumn{2}{c}{ IC 95\% } & p* \\
Adulto mayor & 2,6 & 2,2 & 3,0 & $<0,05$ \\
VIH (+) & 29,0 & 22,7 & 37,0 & $<0,05$ \\
Contacto TBC & 7,4 & 2,8 & 19,8 & $<0,05$ \\
Drogas & 0,8 & 0,5 & 1,2 & 0,285 \\
Extranjero & 2,8 & 2,2 & 3,5 & $<0,05$ \\
Funcionario de salud & 0,8 & 0,4 & 1,7 & 0,579 \\
Situación de calle & 15,6 & 8,1 & 30,1 & $<0,05$ \\
Alcoholismo & 0,5 & 0,3 & 0,7 & $<0,05$ \\
Reo & 15,7 & 11,4 & 21,6 & $<0,05$ \\
\hline Al menos 1 FR & 3,3 & 2,9 & 3,8 & $<0,05$ \\
Sin FR & 0,3 & 0,26 & 0,3 & $<0,05$ \\
\hline
\end{tabular}

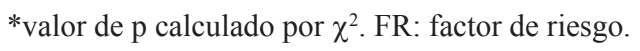

menores tasas que la población de la RM: funcionario de salud, drogadicción y alcoholismo.

La prueba de asociación de $\chi^{2}$ arrojó asociación significativa para adulto mayor, infectado VIH, contacto, extranjero, situación de calle, alcoholismo y reo, todos con $\mathrm{p}<0,05$. Por el contrario, para el uso de drogas $\mathrm{y}$ funcionario de salud, no se encontró asociación $(\mathrm{p}=0,285 \mathrm{y}$ 0,579 respectivamente).

El FR más importante fue el de pertenecer al grupo de los adultos mayores, categoría que se encuentra en casi la mitad de los casos con algún FR y representa el 18,8\% del total de casos nuevos de la RM para el año en estudio.

Los OR encontrados para cada FR variaron entre 0,5 y 29. Para 6 FR se obtuvo un OR significativamente mayor a 1 ; en infectados con VIH se observa el OR más elevado de 29 (IC 95\% 22,737,0 ); reos 15,7 (IC 95\% 11,421,6); situación de calle 15,6 (IC 95\% 8,1-30,1); contactos 7,4 (IC 95\% 2,8-19,8); extranjeros 2,8 (IC 95\% 2,2-3,5); adultos mayores 2,6 (IC 95\% 2,2 -3,0), confirmando el mayor riesgo de enfermar de tuberculosis que tienen los portadores de alguno de estos FR.

Dos grupos presentan OR menores a 1 , pero con un intervalo de confianza que sobrepasa este punto: drogadicción 0,8 (IC 95\% 
0,5-1,2), y funcionario de Salud 0,8 (IC 95\% 0,4$1,7)$, por lo que no se puede establecer con certeza si son factores de riesgo o serían protectores. Finalmente, el alcoholismo presenta un OR 0,5 (IC 95\% 0,3-0,7) (Tabla 5).

\section{Conclusiones}

La identificación de los FR de enfermar de tuberculosis permite focalizar esfuerzos y aumentar la eficiencia de las intervenciones sanitarias. Es de vital importancia conocer el perfil de riesgo y la magnitud de éste en una población. En el nivel de endemia de la tuberculosis en que se encuentra Chile, esto es cada vez más importante para seguir disminuyendo las tasas de incidencia, porque a medida que disminuye el problema, se requieren esfuerzos cada vez mayores para encontrar un caso. El perfil epidemiológico del país en cuanto a tuberculosis concuerda con el de países más desarrollados que el nuestro, con tasas de incidencia cercanas a las de la eliminación avanzada $(<10 / 100.000)$ y riesgo de enfermar que aumenta con la edad de manera importante, con una diferencia hombre/mujer creciente 5 .

No se evidencian diferencias en la proporción de hombres y mujeres entre los casos nuevos y los antes tratados, como tampoco en los promedios de edad entre estos grupos, ni por sexo, lo que podría significar que el riesgo de recaídas y re-infecciones se comportan de manera similar al de la infección reciente.

Se establece de manera clara que el FR más frecuente es ser adulto mayor, que se asocia significativamente a mayor riesgo de enfermar. Otros factores con un riesgo muy alto son la infección VIH, reos y situación de calle. Si bien estos grupos no son tan gravitantes en número como los adultos mayores, sus riesgos son muy superiores, con tasas de incidencia más de 20 veces las del grupo sin FR. Las malas condiciones de vida, desnutrición, hacinamiento, las elevadas tasas de tuberculosis dentro de las cárceles y la inmunodepresión, podrían explicar las magnitudes de riesgo encontrados en este estudio. Estar en contacto con un enfermo contagioso también conlleva un riesgo elevado de enfermar, reforzando la importancia del estudio de contactos en todos y cada uno de los pacientes diagnosticado con tuberculosis pulmonar con baciloscopía positiva.

A pesar de que en general las personas nacidas en el extranjero provienen de países con tasas muy superiores a las de Chile (más de diez veces en países limítrofes) las tasas encontradas dentro de la RM, lugar de concentración del mayor número de extranjeros en el país, sólo son 2,6 veces mayores a la tasa total de la región (OR $2,8)$. Al ser este un grupo que representa tan sólo al 3\% de la población de la RM, no pareciera influir de manera importante en la tasa final de la misma región y del país. Por lo tanto, sería más importante mejorar las condiciones socioeconómicas que hacen que este grupo de personas enferme más de tuberculosis, en vez de intentar identificarlos como potenciales fuentes de brotes epidemiológicos, de manera injustificada y discriminatoria.

No se puede establecer una clara asociación entre ser funcionario de salud o drogadicto y presentar tuberculosis. En el primer caso, por tratarse de un número pequeño de personas, debiera ser analizado en más de un año (un trienio o más) para confirmar las asociaciones descritas en la literatura ${ }^{9}$. En los drogadictos el posible sub-registro de esta condición podría explicar esta falta de asociación, contrario a lo que ha sido descrito para los casos de tuberculosis por infecciones recientes ${ }^{8}$. Situación similar podría explicar el resultado del alcoholismo como supuesto factor protector (OR 0,5 IC95\% 0,3-0,7). A pesar de no existir una clara relación causal para el paso de infección a enfermedad en este grupo $^{17}$, existen evidencias de que sería un FR para la tuberculosis de infección reciente ${ }^{8}$. En la opinión del autor, debiera ser un factor a tomar siempre en cuenta, debido a las condicionantes sociales que acompañan al alcoholismo y el de ser este un factor de mal pronóstico para la adherencia al tratamiento.

A pesar de las limitaciones derivadas de no poder diferenciar si los casos de tuberculosis eran por infección primaria, reactivación endógena o reinfección, como tampoco de poder establecer si los FR conllevan mayor riesgo de exposición al bacilo, de infectarse una vez expuesto a él o de enfermar una vez infectado, es importante conocer la magnitud estimada de riesgo que tienen ciertos grupos, distinguiendo los con riesgo elevado pero presentes en grupos pequeños en número, como las personas en situación de calle, reos o infectados $\mathrm{VIH}$, de aquellos con riesgo menor pero que implican un número importante de casos, como los adultos mayores.

Visualizar diferencias, establecer riesgos e identificar los grupos más vulnerables, permitirá focalizar mejor los recursos, establecer estrategias de mayor eficiencia y reforzar las acciones del PNT, para seguir avanzando hacia la eliminación de la tuberculosis como problema de salud pública. 


\section{Bibliografía}

1.- WHO. Global Tuberculosis Control: Epidemiology, Strategy, Financing. Switzerland, 2009.

2.- FARGA V. La Conquista de la Tuberculosis. Rev Chil Enf Respir; 2004; 20: 101-8.

3.- SENADO J. El riesgo de enfermar de Tuberculosis. Rev Cubana Med Gen Integr 1999; 15: 168-75.

4.- GOBIERNO DE CHILE. Los objetivos de desarrollo del milenio, segundo informe.Santiago, Chile, 2008.

5.- ZUUÑIGA M. La eliminación de la tuberculosis como problema de Salud Pública. Situación en Chile en el año 2008. Rev Chil Enf Respir 2009; 25: 117-26.

6.- CAMINERO J. Guía de la tuberculosis para médicos especialistas. París: Unión Internacional contra la Tuberculosis y Enfermedades Respiratorias, 2003.

7.- MINSAL. Manual de Organización y Normas Técnicas. Programa Nacional de Control de la Tuberculosis. Santiago, Chile. 2005.

8.- NAVA-AGUILERA E, ANDERSSON N, HARRIS E, MITCHELL S. Risk factors associated with recent transmission of tuberculosis: systematic review and meta-analysis. Int J Tuberc Lung Dis 2009; 13: 17-26.

9.- FICA A, RAMONDA P, JEMENAO M I, ZAMBRANO A, CIFUENTES M, FEBRÉ N, et al. Tuberculosis en el personal de salud del Servicio de Salud Metro- politano Sur de Santiago, Chile. Rev Chil Infect 2009; 26: $34-8$.

10.- SECRETARÍA REGIONAL MINISTERIAL DE SALUD REGIÓN METROPOLITANA. Diagnóstico regional participativo: Situación de la epidemia VIH/ SIDA y ETS Región Metropolitana. Santiago, Chile. 2008.

11.- CONASIDA. Panorama epidemiológico de la infección por VIH/SIDA en Chile-Año 2001. Rev Chil Infect, 2002: 19:245-61.

12.- CONACE. Séptimo estudio nacional de drogas en población general de Chile. Santiago, Chile. 2006.

13.- DEPARTAMENTO DE EXTRANJERÍA Y MIGRACIÓN. Estimación de población extranjera en Chile a diciembre 2008. Informe estadístico del Ministerio del Interior, Chile, Santiago, Chile. 2009.

14.- OPS. Salud en las Américas. Volumen II-Países, Washington, 2007.

15.- MIDEPLAN. Catastro Nacional de Personas en Situación de Calle. Santiago, Chile. 2005.

16.- GENDARMERÍA DE CHILE. web oficial: www. gendarmeria.cl. 2010 (último acceso: 10 de Marzo de 2010).

17.- RIEDER H. Bases epidemiológicas del control de la tuberculosis. París: Unión Internacional contra la Tuberculosis y Enfermedades Respiratorias, 1999.

Correspondencia a:

Dr. Christian García C

Programa de Tuberculosis. Ministerio de Salud. Chile.

E-mail: chgarcia@minsal.cl 